\title{
La investigación educativa sobre la formación de biólogos en México: Una mirada a través del tiempo
}

Mtro. Adalberto Aguilar León, Mexicano.

ORCID: https://orcid.org/0000-0001-5742-5972

aleon@uaem.mx

Centro de Investigaciones Biológicas

Universidad Autónoma del Estado de Morelos

Recibido: 18 de agosto del 2020

Aceptado: 22 de septiembre del 2020

\section{Resumen}

El desarrollo de la investigación en el campo de la biología no solo se remite al conocimiento del funcionamiento de la vida o su aplicación para la producción de alimentos, la salud, o el manejo sustentable de los recursos naturales. En paralelo, se ha generado también otro tipo de conocimiento, este último poco conocido, que tiene como propósito originar información confiable para comprender las distintas etapas, a los actores, a las instituciones y el análisis de las prácticas que se desarrollan en los espacios educativos donde se forman los biólogos. El artículo presenta un estado de conocimiento a partir de una revisión bibliográfica de productos de investigación educativa acerca de la formación de biólogos en México a nivel superior, desde su origen a principios del siglo pasado, hasta nuestros días. El trabajo se orientó a reconocer la diversidad de tópicos, intereses, enfoques y tendencias espaciotemporales, además de evidenciar la formación disciplinar y teórica de los autores. Estos elementos se constituyeron como estrategia de comprensión y análisis empírico que permitieron delimitar etapas analíticas que definieron la organización de los resultados. A partir de la construcción de este estado de conocimiento, se expone un panorama general de los tópicos y abordajes metodológicos en las investigaciones con respecto al tema, poniendo en evidencia, a partir de su análisis, un proceso de cambio considerable a través del tiempo, en el que es posible apreciar el tránsito de investigaciones meramente descriptivas, a problemáticas más complejas que permitieron distinguir y delimitar diferentes temáticas y etapas. El proceso de transformación y diversificación de los trabajos aquí mostrados se 
traduce en la posibilidad de reconocer un nuevo campo de investigación que afronta nuevos desafíos para el presente siglo y aporta el primer estado del conocimiento referente a esta temática.

Palabras clave: Investigación educativa, formación del biólogo, carrera de biólogo.

\title{
Education research on the formation of biologists in Mexico: A look through time
}

\begin{abstract}
The development of research in the field of biology not only pertains to the knowledge of the function of life or its application in food production, health, or the sustainable management of natural resources. In parallel, another lesser known type of knowledge has been developed that has the purpose of generating reliable information about the different stages, the actors involved, the institutions and the analysis of the practices that are developed in education institutions where biologists are formed. This study summits a state of knowledge that arises from a bibliographic review of products of investigations on education regarding the formation of biologists in the higher educational levels in Mexico, from its origins in the past century until our current times. The study was oriented into recognizing the diversity of topics, interests, approaches, and space-time tendencies, in addition to the disciplinary formation and theoretical leanings of its authors. These elements were established as a strategy to comprehend and to serve for an empirical analysis that would allow us to delimit analytical stages that defined the organization of the results. From the building of this state of knowledge, a general panorama is exposed of the topics and methodological approaches in the research on the subject, and it puts in evidence from its analysis, a process of profound change through time, from which can be seen the transit from research that is merely descriptive to more complex problems that allows us to distinguish and delimit different themes and stages. The process of transformation and diversification of the research that are shown in this research, show the possibility of recognizing a new field of study that confronts
\end{abstract}


new challenges for the new millennium and contributes the first state of knowledge in this topic.

Key words: Education research, formation of biologists, career in biology.

\section{Introducción}

El desarrollo de la investigación en el campo de la biología no solo se remite al conocimiento del funcionamiento de la vida o su aplicación para la producción de alimentos, la salud, o el manejo sustentable de los recursos naturales. En paralelo, se ha generado también otro tipo de conocimiento, este último, poco conocido, que tiene como propósito originar información confiable para comprender las distintas etapas, a los actores, a las instituciones, así como las prácticas que se desarrollan en los espacios educativos donde se forman los biólogos.

El artículo presenta un estado del conocimiento sobre el desarrollo de la investigación educativa (IE) acerca de la formación de biólogos en México a nivel superior. El trabajo se construyó a partir de una revisión bibliográfica a través del tiempo que puede servir de base para profundizar en las líneas que en él se trabajan, pero también para el análisis de procesos históricos, de transformación de la enseñanza de la biología a nivel superior, o de otros campos.

La investigación recopila los trabajos publicados con respecto a la licenciatura en biología en nuestro país, desde su origen a principios del siglo pasado, hasta nuestros días, otorgando especial énfasis en aquellas investigaciones dadas a conocer en las cuatro últimas décadas. El propósito del presente estudio es distinguir etapas, subrayar su importancia, señalar sus aportaciones e identificar, tanto sus tendencias, como los caminos para fortalecerla en el contexto de las políticas educativas que han tenido lugar en México durante la primera década del siglo XXI y delinear los retos que se tienen que afrontar.

Se documenta la diversidad de tópicos de la IE en este campo y se muestra un

panorama de la transformación, a partir del siglo pasado, de sus intereses, enfoques y tendencias; se pone en evidencia la formación disciplinar y teórica de los autores, así como la manera como ésta ha contribuido a abordar el tema desde muy diversas orientaciones. Se

Revista RedCA octubre 2020 - enero 2021 ISSN: 2594-2824 Vol. 3 Núm. 8 
incluyen trabajos historiográficos de la carrera en nuestro país, sobre el mercado de trabajo de los biólogos, acerca de sus espacios de formación, así como de sus trayectorias escolares y profesionales, hasta arribar a aspectos relacionados con su identidad, sus experiencias y su subjetividad.

Las publicaciones recuperadas permitieron distinguir tres etapas del estado del conocimiento de la investigación educativa desarrollada sobre la formación de biólogos en México; los resultados del estudio se presentan organizados en tres apartados que describen cada una de ellas. El primer apartado da cuenta de los trabajos publicados por los precursores de la biología en nuestro país; sus publicaciones acerca del origen de la carrera ponen en evidencia el nacimiento de la profesión a inicios del siglo pasado. El segundo apartado expone investigaciones donde los profesores, estudiantes y/o egresados de la carrera de biología aparecen como actores principales de la investigación educativa relativa al tema, mientras que el tercero, ubicado a partir del presente siglo, muestra la diversificación en intereses, enfoques y tendencias de los investigadores interesados en la formación de estos profesionales.

El conocimiento de la investigación educativa desarrollada en torno a la profesión permite apreciar que existe una actividad investigadora lo suficientemente relevante como para ser estudiada y desarrollada por una comunidad científica con una formación cada vez más sólida, que toma conciencia de sí misma como campo disciplinar.

\section{Metodología}

Este artículo se constituye como un estado de conocimiento acerca de la formación de profesionales de la biología en nuestro país; su propósito fue contar con un documento analítico, crítico, sistematizado y sintético que, basado en la producción académica, contribuya a comprender la formación de los biólogos mexicanos. La intención de los estados del conocimiento es visualizar perspectivas que orienten el desarrollo futuro de un campo en particular. Lo anterior permite, como asegura Weiss (2003) "identificar los objetos bajo estudio y sus referentes conceptuales, las principales perspectivas teóricas-metodológicas, 
las tendencias y temas abordados, el tipo de producción generada, los problemas de investigación y ausencias, así como su impacto y condiciones de producción” (p. 597).

En ese sentido, Vickery (1970) afirma que este tipo de trabajos se realizan con diferentes propósitos, entre los cuales se encuentran "conocer la producción escrita de otros científicos en un campo específico, acopiar segmentos definidos de información de algún tópico en particular" (p. 154) o, como en este caso, compilar la totalidad de información generada con relación a una temática.

La investigación se deriva de una revisión bibliográfica (Gómez-Luna, Navas, Aponte y Betancourt, 2014) de 86 productos de investigación educativa acerca de la formación de biólogos en México a nivel superior, desde su origen a principios del siglo pasado, hasta nuestros días. En términos de rigurosidad de las investigaciones, el criterio de selección fue su publicación en revistas científicas impresas y electrónicas, presentadas como ponencias en congresos, como tesis de grado y de posgrado, como libros, o como capítulos de libros. Por la misma razón se excluyeron los artículos de divulgación, así como las publicaciones en diarios y periódicos.

La revisión, comparación y comprensión de distintos tipos de fuentes documentales se realizó a través de un abordaje sistematizado y organizado de acuerdo con la propuesta de Peña y Pirela (2007). En este contexto, el trabajo se orientó a reconocer la diversidad de tópicos, intereses, enfoques y tendencias espacio-temporales, además de evidenciar la formación disciplinar y teórica de los autores. Estos elementos se constituyeron como estrategia de comprensión y análisis empírico y permitieron definir etapas para la organización de los resultados.

La delimitación de las etapas permitió confrontar algunos resultados de este trabajo con aquellos derivados de los estados del conocimiento elaborados por el Consejo Mexicano de Investigación Educativa (COMIE) en México. La finalidad de elegir este referente fue la de comparar afinidades y diferencias entre las publicaciones relacionadas con la formación de biólogos y el contexto nacional de la investigación educativa. 


\section{Resultados}

En este estudio se lograron identificar 15 libros, 8 capítulos de libros, 40 artículos publicados en revistas científicas, 12 ponencias en congresos nacionales e internacionales, una tesis de licenciatura, 3 tesis de maestría y 7 tesis de doctorado, rubros considerados para su elección como criterios de rigurosidad.

Los resultados se presentan agrupados en etapas que permiten evidenciar distintos intereses, enfoques e inclinaciones espacio-temporales. Estos periodos se definieron a partir de tres puntos de referencia basados en el número y temática de las publicaciones recuperadas. La primera etapa ubica los trabajos iniciales relacionados con la formación de profesionales de la biología en México; la segunda, ubicada a inicios de los años setenta, da cuenta de un espacio de tiempo que inicia con una mínima producción sobre el tema, para posteriormente, durante los años ochenta y noventa, mostrar un crecimiento exponencial en el número de estas publicaciones. La tercera, ubicada a partir del inicio del presente siglo, se distingue por la diversificación y consolidación de este campo.

\section{Inicios de la biología como profesión en México: los pioneros}

Los primeros trabajos relacionados con la formación de los profesionales de la biología se remontan al año de 1915; uno de ellos, reportado por Cuevas y Ledesma (2006), da cuenta de un fascículo publicado en ese año por Alfonso L. Herrera, a quien se le reconoce por su lucha por establecer los estudios sobre biología en México. Se trata de un boletín difundido por la propia institución denominado "Inauguración de la Dirección de Estudios Biológicos”, instancia que sentó las bases en nuestro país para el estudio y enseñanza de la biología en el siglo pasado. Azuela y Guevara (1998) reportan la existencia de un folleto denominado "La biología en México durante un siglo” dado a conocer en el año de 1921 por el propio Herrera, en donde el autor describe la diferencia en el desarrollo de la biología en nuestro país, antes y después de la revolución mexicana, mientras que, en la Revista de la Sociedad Mexicana de Historia Natural, Herrera López (1939) describe el proceso de constitución de dicha Sociedad, cuyo objetivo fundamental, hasta la fecha, es promover la difusión y publicación 
de los conocimientos que se generan en el campo de las ciencias naturales, así como los resultados originales de investigaciones científicas. Herrera seguirá presente en las publicaciones acerca del desarrollo de la biología en nuestro país, dado que representa el pilar más importante de dicha ciencia en México.

Enrique Beltrán, discípulo destacado de Herrera y considerado como el primer biólogo mexicano escribe "Panorama de la Biología mexicana" y "Consejos a los Biólogos", ambos en el año de 1951; posteriormente, en 1956, "El biólogo en la conservación de los recursos naturales”. Doce años después, en 1968, da a conocer dos artículos en la revista de la Sociedad Mexicana de Historia Natural, el primero en alusión al Centenario de dicha Sociedad y el segundo en honor a su maestro que denominó, “Alfonso L. Herrera (18681968): Primera figura de la biología mexicana". En 1977 publica "Medio siglo de recuerdos de un biólogo mexicano" y finalmente, en 1982, "La contribución de México a la Biología. Pasado, presente y futuro". Estos trabajos componen un invaluable material para comprender el desarrollo de la profesión en nuestro país y constituyen solo una parte de la totalidad de su obra, la cual abordó distintos temas relacionados con el conocimiento de la biodiversidad mexicana.

Hasta el año de 1952, en que la Universidad Autónoma de Nuevo León crea su Facultad de Ciencias Biológicas y oferta la carrera de biólogo, solo era posible acceder a esta profesión en planteles ubicados en la capital de república; la Universidad Nacional Autónoma de México y el Instituto Politécnico Nacional constituían las únicas opciones. Trece años después, en 1965, se apertura un segundo programa educativo fuera de la zona metropolitana de la Ciudad de México en la Universidad Autónoma del Estado de Morelos, iniciando la multiplicación de la carrera en otras entidades del país, acompañada de un aumento considerable de la matrícula de biólogos en formación en México.

La figura de Alfonso L. Herrera resulta fundamental para comprender el desarrollo de la biología mexicana; aún más, parafraseando a Ledesma (2013), se trata del personaje que da identidad a la profesión. Su figura, indiscutiblemente, permanece hasta nuestros días 
en el escenario de las publicaciones que, desde diversas perspectivas, pretenden comprender el avance y transformación de esta disciplina en México.

\section{Investigación educativa sobre la formación de biólogos en nuestro país: el vacío y el despegue}

Al inicio de los años setenta, era posible apreciar que los trabajos publicados por Herrera y posteriormente por Beltrán, constituían las pocas aportaciones al tema de la formación de biólogos en nuestro país, destacándose, luego de un largo vacío, el trabajo de Betancourt (1974), quien pondera la importancia de la implementación del aula-laboratorio en las nuevas instalaciones de la Facultad de Ciencias de la UNAM, con el fin de agilizar la relación entre la clase teórica y la clase práctica. Un año después, Toledo (1975), bajo un enfoque sumamente crítico publica "Las 4 Biologías de una universidad subdesarrollada: La UNAM", mientras que un artículo elaborado por Cifuentes (1975) da cuenta de "La biología en la Facultad de Ciencias", desarrollado en la misma institución.

Durante la década de los ochenta se dan a conocer los primeros trabajos en los que los profesores, estudiantes y/o egresados de la carrera de biología en nuestro país aparecen como sujetos de investigación educativa; sus rasgos corresponden a aquellos que definieron las tendencias en el nivel superior en dicha etapa, caracterizada como un área emergente en proceso de desarrollo. Para ese periodo, Colina (2010) encuentra que, en el ámbito de la investigación educativa en México, publican sus primeras investigaciones alrededor de noventa investigadores, la mayoría de ellos, adscritos a instituciones académicas ubicadas en la zona metropolitana de la Ciudad de México.

En ese mismo sentido, Barrón e Ysunza (2003) señalan, para esa misma época en México, que las investigaciones que abordaron el tema del currículum de nivel superior en particular, lo hicieron en relación con la problemática de las profesiones y la búsqueda de modelos alternativos para la formación de los estudiantes universitarios (intentos de evaluación y reestructuración de planes y programas de estudio, o análisis de los contenidos curriculares), como ejemplo de ello encontramos el trabajo de Foliari y Soms (1981), quienes 
publican una crítica al modelo teórico de la departamentalización. La mayoría de esos trabajos correspondieron a tesis de grado y posgrado o a documentos generados al interior de las IES (Instituciones de Educación Superior) y, como afirman Barrón e Ysunza (2003), éstos se realizaron desde diferentes perspectivas, entre las que destacan la del conocimiento disciplinario propio de un proyecto curricular, el enfoque de las prácticas laborales y el mercado ocupacional, la transformación histórica de las profesiones, la sociología del conocimiento y el estudio de los procesos de aprendizaje, así como la apropiación de contenidos curriculares y habilidades profesionales en el estudiante de nivel superior.

En coincidencia con el panorama de la investigación educativa a nivel nacional, en este periodo es posible observar que la mayor parte de las publicaciones relacionadas con la licenciatura en biología, fueron dirigidas a conocer el ámbito profesional de los biólogos, utilizando, esencialmente, métodos cuantitativos que daban cuenta de las condiciones y espacios de trabajo en donde éstos se ubicaban laboralmente. Los primeros antecedentes de esta tendencia se encuentran desde finales de los años sesenta y lo constituyen trabajos como el de Barrera en 1968 denominado "Panorama de la Biología en México", así como el de Páez (1970) titulado "Estudio del Mercado de Trabajo del Biólogo".

En la década de los ochenta se manifiesta claramente un interés en el tema del trabajo de los egresados. Como ejemplo de ello, encontramos las investigaciones de López de la Rosa desarrolladas entre 1984 y 1987. Gleason, López de la Rosa y Mendoza publican en 1983 "Diagnóstico preliminar sobre las actividades profesionales que desarrolla el biólogo en la Ciudad Universitaria, D.F."; López de la Rosa da a conocer en 1984 "Las actividades profesionales del biólogo en La Paz, Baja California Sur", posteriormente, en 1985 "Actividades profesionales del biólogo en el Instituto Nacional de Investigaciones sobre Recursos Bióticos, en sus sedes de Jalapa y el Distrito Federal”, en 1986, junto a Ríos y Mendoza "Elementos para el seguimiento académico en la carrera de Biología de la Facultad de Ciencias de la UNAM” para finalmente, en 1987, “Actividad profesional del Biólogo en el Instituto Tecnológico y de Estudios Superiores de Monterrey y en la Facultad de Ciencias de la Universidad Autónoma de México". Al mismo tiempo, y en esa misma línea, Méndez 
(1985) publica "Las actividades profesionales del biólogo en diversas dependencias de la Secretaria de Agricultura y Recursos Hidráulicos". Al mismo tiempo y retomado el tema de los orígenes de la profesión, Gaxiola desarrolla una tesis de licenciatura para titularse como biólogo con el tema "Historia de la biología en México en el siglo XX: La obra de Enrique Beltrán".

Es precisamente en una de las investigaciones citadas, donde se hace referencia a la existencia de un laboratorio de investigación educativa ubicado en la Facultad de Ciencias de la Universidad Nacional Autónoma de México (Ríos, López de la Rosa y Mendoza, 1983), dato que muestra, para esa época, el interés institucional por desarrollar proyectos encaminados a mejorar, a través de la investigación científica, la manera como se formaban los profesionales de esta disciplina. En contraste, hasta ese momento, las prácticas, tanto de los profesores, como de los estudiantes, siguen estando ausentes como campos de investigación.

Otra carencia evidente se refleja en el mínimo número de investigaciones desarrolladas sobre el tema del currículum de la carrera; Leal (1985) obtiene el grado de Maestra en Educación Superior con la tesis denominada "Propuesta metodológica para la evaluación del plan de estudios de la carrera de biólogo en la Facultad de Ciencias Biológicas de la Universidad Autónoma de Nuevo León”, investigación que se constituye como uno de los pocos ejemplos del interés por el campo.

La década de los ochenta se caracterizó también, como afirman Noriega, Lagunas y Recio (2009) por un profundo cambio en las relaciones entre el Estado y las IES, a través de políticas de evaluación que determinaron el crecimiento y las orientaciones de las universidades públicas del país. Las universidades buscaron construir estrategias para conseguir los indicadores requeridos por el gobierno federal que impactaron, en consecuencia, a los aproximadamente veinte programas educativos de la carrera de biólogo existentes en México. Estas presiones se reflejan, como puede apreciarse, en los tópicos de interés abordados en la investigación educativa desarrollada sobre la carrera en ese periodo. 
Con respecto a los estudiantes y profesores universitarios como tema central de investigación, Carvajal, Spitzer, Guzmán y Zorrilla, (1996) sostienen, para el primer caso, que el estudiante, pese a ser el protagonista del proceso de enseñanza aprendizaje, era un sujeto desconocido del que poco se sabía. Aun cuando los esfuerzos educativos de la época eran dirigidos hacia ellos, paradójicamente se mostraba poco interés en investigarlos, afirmación que coincide con lo encontrado en la producción científica acerca de la licenciatura en biología. En contraste, los estudios reportados por Ducoing y Landesmann (1996) muestran el naciente interés por conocer, bajo diferentes perspectivas, el trabajo de los académicos, lo que no ocurre con las investigaciones tendientes a analizar su papel como formadores de biólogos.

En relación con la manera de abordar este tipo de trabajos, es posible apreciar, en el contexto de la investigación educativa a nivel nacional, el predominio de estudios de tipo cuantitativo que enfocaban a los estudiantes como parte de la matrícula o de la población escolarizada, y aquellos de tipo psicológico, especialmente los que se centraban en procesos de aprendizaje, sin embargo, estos enfoques no se ven reflejados en las publicaciones sobre la carrera de biólogo de la época. Como es factible distinguir, en los años ochenta estas investigaciones se limitan a dar cuenta de la actividad profesional del biólogo y no corresponden a las tendencias de la década, debido, posiblemente, a que constituían temas de interés para las instituciones que ofertaban la carrera, o bien, por la carencia de académicos con la formación necesaria para poder desarrollarlos al interior de los programas educativos.

En los años noventa existían poco más de cuarenta programas educativos que formaban biólogos en nuestro país, todos ellos listados en el "Directorio de Facultades, Escuelas e Institutos que imparten la carrera de Biología a en la República Mexicana” publicado en el año de 1991 por la Asociación Mexicana de Facultades y Escuelas de Biología (AMFEB). Este documento muestra un crecimiento de más del 100\% en solo una década en el número de IES que ofertaban la licenciatura en nuestro territorio, incluidos dos programas ubicados en universidades privadas. 
Durante ese lapso las IES seguían presionadas por el gobierno federal a través de la implantación de políticas que intentaban responder a las exigencias de la globalización y tendientes a la búsqueda de la calidad y la competitividad. Estas directrices propiciaron, a diferencia de la década anterior, un acelerado crecimiento de la planta docente, particularmente en las universidades estatales con ayuda del denominado Programa de Mejoramiento del Profesorado (PROMEP), que les permitieron diversificar sus opciones educativas, pero también sus áreas de investigación. El arribo de investigadores en el área educativa no fue la excepción, aunque en el caso de la formación de biólogos, aún no es posible apreciar con claridad, en esa etapa en particular, su impacto en el número y diversidad de publicaciones relacionadas con la formación de estos profesionistas.

En los años noventa, la tendencia a desarrollar investigaciones sobre la ubicación profesional de los egresados continúa, aunque ahora dedicando un mayor interés a conocer los espacios universitarios en donde se forman los biólogos en México, así como las temáticas para lograr la titulación profesional. Como ejemplo de estos trabajos encontramos títulos como el de Ledesma (1990) "Esbozo del desarrollo histórico de la biología en Puebla" en 1992, "Revisión preliminar del proceso de titulación de los egresados de la carrera de Biología de la ENEP-I UNAM de Granillo y colaboradores y bajo la misma vertiente, "Evaluación de la enseñanza de la Biología en México" de Tirado y López Trujillo en 1994, mientras que en el año de 1999 se publica "La licenciatura en Biología en la ENEP - Iztacala de la UNAM" de López Trujillo y Saucedo. Tópicos como la comprensión de los estudiantes de conceptos fundamentales para la disciplina, entre los que destaca el de selección natural, son también del interés de autores como Millán y colaboradores en el año de 1997.

El desarrollo histórico de los espacios de formación de los biólogos en nuestro país se constituye también, en la década de los noventa, en tema de interés de los investigadores, lo que se percibe desde finales de los años ochenta con los trabajos de Rodríguez (1987) quien escribe "La educación superior de la biología en México" y de Cifuentes (1989), que produce "La Biología en la Facultad de Ciencias de la UNAM". Es así como Hoffmann, Cifuentes y Llorente (1993) publican "Historia del Departamento de Biología de la Facultad 
de Ciencias, UNAM, México", Islas (1993) "Pasado y presente en la formación profesional del biólogo", y Ledesma, en el mismo año, "Biología: ¿ciencia o naturalismo?”; Ortega, Godínez y Vilaclara (1996) desarrollan el tema "Relación histórica de los antecedentes y orígenes del Instituto de Biología de la Universidad Autónoma de México" y finalmente, Domínguez (1999), "La creación de la Facultad de Ciencias".

Como puede apreciarse, durante la década de los noventa, el número de publicaciones, así como la diversidad de temas referentes a la formación de biólogos en México empieza a diversificarse. Sin embrago, es a partir del año 2000 cuando se advierte un desarrollo importante en el abordaje de los estudios sobre la carrera de biología en nuestro país, ya que da inicio la producción de trabajos basados en metodologías cualitativas provenientes de la investigación educativa y realizados por profesionales de otras disciplinas, así como por biólogos con formación especializada en este campo. Para este periodo, es posible advertir, en ese sentido, el impacto de las contrataciones de nuevos investigadores por parte de las IES, así como el creciente interés de los propios biólogos por incursionar en el tema de la formación de sus pares, o como resultado de su ingreso, como estudiantes, a posgrados de educación.

\section{El siglo XXI: la consolidación}

En el presente siglo aparecen investigaciones que dan cuenta de las transformaciones históricas y filosóficas experimentadas por la biología como profesión; así lo muestran trabajos como el de Barahona, Suárez y Martínez (2001) denominado "Filosofía e Historia de la Biología", el de Barahona y Ledesma (2002) titulado "El positivismo y los orígenes de la biología en México" o el de Ledesma (2009) "Biología, institución y profesión: centros y periferias" libro en el cual, para el caso de México, se analizan las tesis profesionales producidas por los biólogos en nuestro país entre 1947 y 1978. Bajo este esquema, el mismo autor, pero en el año 2013, publica "La Biología y los biólogos en México: ciencia, disciplina y profesión”. 
En torno al currículum para la formación de biólogos, en el presente siglo destacan los trabajos de Lugo (ambos en el año 2003), investigadora educativa quien realiza estudios comparativos entre modelos curriculares flexibles en programas de licenciatura en universidades públicas mexicanas. Sus investigaciones identifican los principales elementos académicos y administrativos que se pusieron en operación al incursionar en ese tipo de modelos. La autora caracteriza sus componentes y plantea propuestas respecto a las condiciones ideales que apoyen su instrumentación. Los estudios de Lugo incluyen los programas educativos homólogos de la Universidad Autónoma del Estado de Morelos, de la Universidad de Guadalajara (U de G), de la Benemérita Universidad Autónoma de Puebla (BUAP) y de la Universidad Autónoma de Baja California (UABC).

Otros trabajos sobre currículum de la profesión son el de Pino (2008), quien se aboca al análisis de la relación entre docencia e investigación en la Facultad de Ciencias de la UNAM, así como el de Ordóñez (2009), quien realiza una investigación en tres licenciaturas de la Universidad Juárez Autónoma de Tabasco (UJAT), entre las que se encuentra la carrera de Biología; en dicho trabajo se destacan los roles de participación, tanto de profesores, como de los estudiantes.

Aguilar y Aranda (2009) publican una investigación dirigida a conocer la opinión de los profesores de la licenciatura en sobre el modelo curricular flexible de la licenciatura de Biología de la Facultad de Ciencias Biológicas (FCB) de la Universidad Autónoma de Estado de Morelos (UAEM), mientras que Monterrosas desarrolla, como temas de tesis, primero de Maestría (2010), "La tutoría como un dispositivo que integre perspectivas de estudiantes, docentes, directivos, currícula y gestión educativa" y posteriormente, en el 2018, de Doctorado "Establecimiento del vínculo tutor-tutorado y su repercusión en el proceso de formación profesional de los estudiantes", ambos trabajos en el contexto del mismo programa educativo. Con respecto a los contenidos de los planes de estudio Aguilar-Aguilar (2015) aborda el tema de la elección de alguna actividad principal por parte de los futuros biólogos, así como las motivaciones y orientaciones diversas que influyen en dicha elección. 
Como puede apreciarse, los trabajos publicados acerca del currículum de esta carrera se dirigen primordialmente a los actores del proceso enseñanza-aprendizaje. Los estudiantes y profesores son el punto nodal de interés de los autores, quienes incursionan en nuevas líneas de investigación; al mismo tiempo, su revisión permite distinguir que, tanto su formación profesional, como las líneas de investigación que desarrollan, son sumamente diversas.

La oferta educativa de la carrera de Biología en México es también revisada desde otros enfoques; es así como López Trujillo desarrolla su tesis doctoral en el año 2009 con el tema "La Licenciatura de Biología en México", que da cuenta detallada de los programas educativos distribuidos en el territorio nacional, en tanto que Sigala, Barba, Meza, Castorena y González (2014), examinan la trayectoria histórica de la licenciatura en la Universidad Autónoma de Aguascalientes, describiendo la manera como sus egresados se han insertado en la sociedad.

Los procesos de evaluación y acreditación de los programas educativos de nivel superior en nuestro país también han influido en despertar el interés de las IES por abordar nuevas temáticas de investigación al interior de las universidades mexicanas. Como consecuencia, desde el inicio del presente siglo, es posible encontrar investigaciones encaminadas a evaluar el aprendizaje de conceptos esenciales para la formación del biólogo y que muestran cómo la historia y la epistemología de la ciencia pueden ser aprovechadas para definir enfoques didácticos que favorezcan la comprensión de las teorías científicas (Hernández y Ruiz, 2000), o sobre la forma en que los estudiantes construyen conocimiento en el aula (Campos, Gaspar y Alucema, 2000), del mismo modo, trabajos dirigidos a la exploración de aspectos curriculares, en particular en lo tocante a la relación entre la investigación científica y su impacto en la docencia (Campos, 2002).

El desarrollo de los procesos de aprendizaje de los futuros biólogos, a partir del trabajo de los profesores en las aulas, es también motivo de interés de varios investigadores desde los inicios del siglo XXI; Eisenberg, Olivares, Duarte y González Díaz, (2004) evalúan los resultados de la implementación de un curso monográfico en la Facultad de Educación 
Superior-Iztacala (FES-I) de la Universidad Nacional Autónoma de México (UNAM), cuyo objetivo consistía en preparar a los futuros biólogos para el manejo del conflicto en escenarios laborales reales. Rebolledo y Ayala (2006) evalúan su desempeño docente, en tanto que Torres (2002), valora el impacto de la investigación científica en su formación, y posteriormente, en el año 2011, utiliza el Modelo de Análisis Proposicional (MAP) para establecer comparaciones cualitativas de aproximación de significado, con la finalidad de comprender el aprendizaje del conocimiento generado por la investigación científica en estudiantes de un curso regular en una universidad pública.

Bajo esa misma lógica, en este siglo se destacan demandas institucionales específicas de información referente a trayectorias y éxito profesional de los egresados, retomándose así los temas con que inician los estudios sobre estudiantes de biología en la década de los ochenta. Trabajos como el de Jiménez (2009), analizan las trayectorias de los estudiantes de esta licenciatura a partir de lo que el autor llama un método retrospectivo y transversal para la reconstrucción de los itinerarios laborales de los egresados, pero basándose en el concepto sociológico de Boado (1996). En este mismo ámbito encontramos los trabajos de Rebolledo, Gómez del Prado y Romero (2005), quienes caracterizan a los estudiantes de Biología Marina de la UABCS, así como el de Romero y colaboradores (2007) acerca de las trayectorias de los estudiantes de la misma licenciatura y su relación con el nivel medio superior; para este mismo programa educativo, Rebolledo y Romero (2011) desarrollan una investigación que pone en evidencia la relación entre las características de los jóvenes al ingreso y su trayectoria escolar. Jiménez y Orozco (2011) analizan la inserción y el desempeño profesional de los biólogos agropecuarios graduados de la Universidad Autónoma de Tlaxcala desde la perspectiva de los empleadores y los propios egresados, mientras que Aragón y Chávez (2009) investigan el perfil de personalidad del estudiante de Biología en la FES-I de la UNAM, describiéndolos como poseedores de alta inteligencia y capacidad mental escolar que, bien encauzadas, les augura éxito, tanto en la terminación de sus estudios, como en su desenvolvimiento laboral. 
Pese a la diversificación en intereses, enfoques y tendencias observadas en las investigaciones desplegadas en el campo de la educación superior en el presente siglo, para el campo particular de la formación de biólogos en México, los procesos históricos y filosóficos implicados en dicho proceso, así como el desarrollo y transformación de la carrera, se mantienen como principales tópicos de interés de los investigadores. Al mismo tiempo, es posible apreciar mayor profundidad en los trabajos, así como un notable incremento en la complejidad de las temáticas abordadas.

Desde finales del siglo anterior (1999), Aullet publica "Alfonso L. Herrera y la Dirección de Estudios Biológicos. Trascendencia y actualidad de un proyecto nacionalista para el desarrollo científico y tecnológico"; en el mismo año Ledesma desarrolla sus Tesis doctoral con el tema "El conflicto entre Alfonso L. Herrera e Isaac Ochoterena y la institucionalización de la biología en México.” y meses después, junto a Barahona, publica “Alfonso Luis Herrera e Isaac Ochoterena: la institucionalización de la Biología en México”.

Ledesma, reconocido por su intensa producción en el campo publica, en el año 2002, "La introducción de los paradigmas de la biología en México y la obra de Alfonso L. Herrera"; en 2003, junto con Barahona, "The Institutionalization of Biology in Mexico in the early 20 th Century. The Conflict Between Alfonso Luis Herrera (1868-1942) and Isaac Ochoterena (1885-1950)" y posteriormente, en 2006, “Alfonso L. Herrera: controversias y debates durante el inicio de la biología en México”. Cuevas y el propio Ledesma, en el mismo año, divulgan “Alfonso L. Herrera: controversia y debates durante el inicio de la biología en México”. En 2007 Ledesma escribe "De Balderas a la Casa del Lago. La institucionalización de la Biología en México”, obra publicada por la UNAM.

De la Paz contribuye al tema en el año 2010 con la Tesis de Maestría denominada “De la historia natural a la profesionalización de la Biología en México (1900-1940)” para posteriormente publicar, en el 2012, “Cómo surgió la Biología como profesión?”. Cuevas, por su parte, da a conocer en el 2011 "La biología en México: el origen de una profesión” y recientemente, en el año 2018, “La enseñanza de la biología en México entre 1896 y 1908 , 
un estudio de caso". Como he señalado y dada su importancia, la presencia de Herrera en las publicaciones sobre la formación de biólogos en nuestro país continua presente hasta nuestros días, recibiendo el reconocimiento como precursor de esta disciplina.

Durante la segunda década del presente siglo, resulta notable la diversificación de las investigaciones acerca de la profesión iniciada en el año 2000; Urquidi (2011) encuentra, en la Universidad Veracruzana (UV), que la población estudiantil de la carrera es poseedora de un mayor capital cultural de origen que los estudiantes de contaduría, mientras que MatusParada (2011) explorara las deficiencias del aprendizaje de una actividad compleja (diagnóstico ecológico) en alumnos de la Universidad Autónoma Metropolitana (UAM) Xochimilco y desarrolla una propuesta de modelo didáctico. En tanto, Arriaga, Gracian, Muñoz, Mandujano y Cruz (2014) ponderan el valor didáctico del diagnóstico en general, como herramienta inductora a un pensamiento relacional en estudiantes de ese mismo programa educativo. En el año 2013, López Trujillo, Nava y Moreno exploran los estilos de aprendizaje en los estudiantes de la carrera de Biología en la ENEP-I y Reyes Lazalde, Reyes y Pérez, en 2016, desarrollan en la Universidad de Guadalajara el trabajo denominado "Experimentación virtual con el simulador dosis-respuesta como herramienta docente en biología". Estas investigaciones no solo diversifican los temas abordados, suponen también un nuevo camino hacia la comprensión del proceso de formación de biólogos y no se limitan únicamente a su descripción.

En el año 2015 la FES-Iztacala de la UNAM publica el libro "la Biología y los biólogos en México. Historia y representaciones sociales" resultado de los avances en toda una línea de investigación desarrollada en esa unidad académica y que contiene cinco trabajos, los cuales pretenden, como señalan sus autores y partiendo de la distinción entre ciencia y profesión, aunar a la visión histórica de una ciencia con una dimensión social. Los trabajos agrupados en este libro son "Influencias de la elección de una profesión: biólogo y médico" de Contreras, "La segunda opción como perversión de la vocación científica: el caso de la carrera de biólogo" de Contreras y Ledesma, Los biólogo: representación social de un grupo sociocultural" de Contreras y Tlacomulco, "Entre lo sociológico y lo histórico: 
Elementos de la representación social de la evolución entre estudiantes de Biología, medicina y Psicología" de Medina y Ledesma y finalmente "Representación social del Modelo de desarrollo entre biólogos y carreras afines al medio ambiente” de Fernández y Lara.

Los trabajos referidos permiten visualizar, para esta etapa, la consolidación de una línea de investigación relacionada con la formación de biólogos en nuestro país; al mismo tiempo, proporcionan elementos para apreciar el crecimiento, desarrollo, profundización y diversificación de las temáticas a desarrollar en la siguiente década.

\section{Conclusiones}

El estado del conocimiento expuesto en este trabajo muestra un panorama general de los temas y abordajes metodológicos encontrados en las investigaciones relacionadas con los programas educativos de la carrera y la formación de biólogos en nuestro país, lo que hace posible distinguir un proceso de cambio considerable a través del tiempo, transitando de análisis meramente descriptivos, a los comprensivos. Esta situación no representa un caso particular de la profesión, o único en México; la investigación educativa realizada en otros programas de la licenciatura en nuestro territorio se muestra determinada por racionalidades e intereses diversos de legitimidad, así como a etapas de los procesos investigativos enfocados a la educación superior.

El análisis de la diversidad de tópicos, intereses, enfoques y tendencias espaciotemporales, así como la formación disciplinar y teórica de los autores de los trabajos recuperados en este trabajo, permitieron reconocer y delimitar etapas de producción de la investigación educativa sobre el campo y compararlos con las tendencias de la investigación educativa a nivel nacional, tal como se muestra en los resultados. En una primera etapa, durante la primera mitad del siglo pasado, los pioneros se interesaron por mostrar a la sociedad aspectos históricos del nacimiento formal de la profesión, así como la lucha por establecer los estudios sobre biología en México, dando a conocer los distintos espacios de participación del biólogo. Los "pioneros" siguen presentes en el debate hasta hoy en día, 
reconociéndoles su importante labor, ya que abonaron en el proceso de institucionalización de la carrera en términos de instancias, tanto de investigación, como de docencia.

Más tarde, a partir de los años setenta y ochenta, y luego de un prolongado vacío, la actividad profesional del biólogo, a partir de investigaciones de tipo cuantitativo, se convierte en tema recurrente, a diferencia de lo que ocurría en otros campos de la investigación educativa en los que se abordaban múltiples aspectos. Resulta notorio que estos trabajos, desarrollados comúnmente por biólogos, obedecían a las necesidades propias de la época, entre las que se encontraba asegurar la matrícula de los PE de biología y demostrar la importancia de la profesión.

En contraste, en los años noventa, el crecimiento y diversificación de la planta docente, especialmente en las universidades públicas estatales, propició el abordaje de múltiples problemáticas detectadas en los procesos de formación profesional de los biólogos, lo que se reflejó en las investigaciones reportadas. Este proceso de desarrollo académico y despunte de los trabajos publicados con respecto al campo, impulsado también por las exigencias de los procesos de evaluación y acreditación de las IES, se observa con claridad a partir del presente siglo; es entonces cuando estudiosos de otras disciplinas se acercan a al tema de la formación de biólogos.

El artículo permite apreciar que los principales interesados en estudiar la formación de los biólogos en México han sido los propios biólogos, algunos de los cuales se han formado académicamente en otras disciplinas para incursionar en diversos temas relacionados con la historia, la sociología, la filosofía o la investigación educativa. Sin embargo, investigadores con formaciones muy diversas ha realizado importantes contribuciones, diversificando y profundizando en temas y enfoques antes poco estudiados.

Las 86 publicaciones recuperadas en este estudio componen el primer estado del conocimiento sobre el tema de la formación de biólogos en México y proporciona elementos de reflexión interna sobre la propia disciplina. El proceso de transformación y diversificación de los trabajos aquí mostrados se traduce en la posibilidad de reconocer un nuevo campo de 
investigación que afronta nuevos desafíos para el presente siglo. El reto lo constituye el diversificar y profundizar aún más en las líneas de investigación desarrolladas, detectar nuevos objetos de estudio y actualizar el conocimiento generado, con el fin de impactar en la orientación de los procesos de enseñanza-aprendizaje y en general en la formación de biólogos en nuestro país.

La intención de este artículo, como sostiene Jiménez (2006), es que la investigación de la investigación, como producto del presente, sirva como conocimiento acumulado para iniciar nuevas reflexiones.

\section{Referencias bibliográficas}

AMFEB-CNES, (1991). Directorio de Facultades, Escuelas e Institutos que Imparten la Carrera de Biología en la República Mexicana. México: Asociación Mexicana de Facultades y Escuelas de Biología A.C. y Consejo Nacional Para la Enseñanza de la Biología A.C. México. D.F.

Aguilar, A. R. (2015). ¿Tú que tipo de biólogo quieres ser? Ciencias, núm. 115-116, enerojunio, pp. 84-92. https://www.revistaciencias.unam.mx/es/199-revistas/revistaciencias-115-116/1917-\%C2\%BFt\%C3\%BA-qu\%C3\%A9-tipo-de-bi\%C3\%B3logoquieres-ser.html.pdf

Aguilar, L. A. y Aranda, O. R. (2009). El tránsito hacia un currículo flexible desde el punto de vista del personal académico. El caso de la carrera de biólogo en la Universidad Autónoma del Estado de Morelos. En R. López Zárate (Presidente) X Congreso Nacional de Investigación Educativa. Recuperado de http://xplora.ajusco.upn.mx:8080/xplora-pdf/0508-F.pdf

Aragón, B. L., y Chávez, L. R. (2009). Perfil de personalidad del estudiante de biología de la FES Iztacala UNAM. En R. López Zárate (Presidente), X Congreso Nacional de Investigación Educativa. COMIE, Veracruz, México.

Arriaga F. A., Gracian S. S., Muñoz V. A. L., Mandujano, P. M., y Honorato de la Cruz, G. G. (2014). El diagnóstico como estrategia para la inducción del pensamiento relacional en la carrera de Biología. Revista de la Educación Superior, 43(169), 107124.

Aullet, G. (1999). Alfonso L. Herrera y la Dirección de Estudios Biológicos. Trascendencia y actualidad de un proyecto nacionalista para el desarrollo científico y tecnológico. Memoria del Primer Congreso Mexicano para el Avance de la Ciencia y la

Revista RedCA octubre 2020 - enero 2021 ISSN: 2594-2824 Vol. 3 Núm. 8 
Tecnología, México, Sociedad Mexicana para el Progreso de la Ciencia y la Tecnología. 375.

Azuela, L. F. y Guevara, F. R. (1998). La ciencia en México en el siglo XIX. Una aproximación historiográfica. Asclepio. Madrid. L (2), 5-33.

Barahona, E. A. y Ledesma, M. I. (2002). El positivismo y los orígenes de la biología en México: El entrelazado de los desarrollos filosófico y científico en un contexto histórico particular. Archives internationales d'histoire des sciences, 52(149), 277305.

Barahona, E. A., Suárez, E. y Martínez, S. (Compiladores). (2001). Filosofía e Historia de la Biología. Universidad Nacional Autónoma de México.

Barrera, A. y Laguna, J. (1968). Panorama de la biología en México, México, Centro Nacional de Productividad. México, D.F.

Barrón, C. e Ysunza, M. (2003). Currículo y formación profesional en Díaz Barriga, A. (coord.), La investigación curricular en México. La década de los noventa, México, Consejo Mexicano de Investigación Educativa, pp. 125-164.

Beltrán, E. (1951). El panorama de la biología mexicana. Revista de la Sociedad Mexicana de Historia Natural. 12, 23- 30. México.

Beltrán, E. (1951). Consejos a los biólogos, normas y métodos para la investigación biológica). Ediciones del Instituto de Investigación Científica de la Universidad de Nuevo león, (No. 574.07 B4). Monterrey, México. 173 pp.

Beltrán, E. (1956) El biólogo en la conservación de los recursos naturales. Instituto Mexicano de Recursos Naturales Renovables. México. 1,53

Beltrán, E. (1977). Medio siglo de recuerdos de un biólogo mexicano. México. Sociedad Mexicana de Historia Natural. México. 493.

Beltrán, E. (1968a). El primer centenario de la Sociedad Mexicana de Historia Natural. Revista de la Sociedad Mexicana de Historia Natural. México. 29, 119- 120.

Beltrán, E. (1968b). Alfonso L. Herrera (1868-1968). Primera figura de la biología mexicana. Revista de la Sociedad Mexicana de Historia Natural. México. 29, 38.

Beltrán, E. (1982). Contribución de México a la biología. Pasado, presente y futuro. Consejo Nacional para la Enseñanza de la Biología, AC. Editorial Continental, Distribuidores. México. 121 pp.

Betancourt, J. (1974). Una nueva escuela de biología. Revista Biología, CNEB, 3(4).

Revista RedCA octubre 2020-enero 2021 ISSN: 2594-2824 Vol. 3 Núm. 8 
Boado, M. (1996). Movilidad ocupacional y mercado de trabajo: las caras ocultas del empleo urbano en Montevideo. Revista de Ciencias Sociales. Uruguay. 12, 1-14.

Campos, M. A. (2002) La investigación Científica en la Biología y su impacto en la docencia universitaria. El caso de la universidad Michoacana. (Tesis Doctoral). ENEP Aragón. Universidad Nacional Autónoma de México.

Campos, M. A., Gaspar, S., y Alucema, A. (2000). Análisis de discurso de la conceptualización de estudiantes de biología de nivel universitario. SOCIOTAM: Revista Interdisciplinaria de Ciencias Sociales y Humanidades, 10 (1), 31-71.

Carvajal, A., Spitzer, T., Guzmán, C., y Zorrilla, J. F. (1996). Alumnos. estado de conocimiento. En P. Ducoing y M. Landesman (coords.), Sujetos de la educación y formación docente (pp. 27-81). D.F., Consejo Mexicano de Investigación Educativa, México. 27-81.

Cifuentes, L. J. L. (1975) La Biología en la Facultad de Ciencias. Revista de la Sociedad Mexicana de Historia Natural. México. 36, 7-24.

Cifuentes, L. J. L. (1989). La Biología en la Facultad de Ciencias de la UNAM. En Primer Congreso Mexicano de Historia de la Ciencia y la Tecnología, Tomo I (pp. 196 203), México.

Colina, E. A. (2010). El crecimiento del campo de la investigación educativa en México. Un análisis a través de sus agentes. Perfiles educativos, México. 33(132), 8-26.

Contreras, A. M. (2015). Influencias en la elección de una profesión: biólogo y médico. En M. I. Ledezma y A. M. Contreras (Comps.), La Biología y los biólogos en México. Historia y representaciones sociales $\left(1^{\mathrm{a}}\right.$ ed.) (pp. 23-54). D.F., México: Universidad Nacional Autónoma de México.

Contreras, A. M., y Ledezma, M. I. (2015). La segunda opción como perversión de la vocación científica: el caso de la carrera de biólogo. En M. I. Ledezma y A. M. Contreras (Comps.), La Biología y los biólogos en México. Historia y representaciones sociales ( $1^{a}$ ed.) (pp. 55-72). D.F., México: Universidad Nacional Autónoma de México.

Contreras, A. M. y Tlacomulco, M. C. (2015). Los biólogos: representación social de un grupo socioprofesional. En M. I. Ledezma y A. M. Contreras (Comps.), La Biología y los biólogos en México. Historia y representaciones sociales ( $\left.1^{\mathrm{a}} \mathrm{ed}.\right)$ (pp. 73-90). D.F., México: Universidad Nacional Autónoma de México.

Cuevas, C. M. del C. (2011) La biología en México: el origen de una profesión. Revista Ciencia Universitaria. UAEH. México. 1. (2), 73-82.

Revista RedCA octubre 2020 - enero 2021 ISSN: 2594-2824 Vol. 3 Núm. 8 
Cuevas, C. M. del C., y Ledesma M. I. (2006). Alfonso Herrera: Controversia y Debates. Rev. Historia Mexicana. El Colegio de México. 3 (55), 973-1013.

Cuevas, C. M del C. (2018). La enseñanza de la biología en México entre 1896 y 1908, un estudio de caso. Revista de historia de las ciencias y las humanidades, HCH, A.C. México. 1, (3) 101-116.

Cuevas, C. M. del C. y Ledesma, M. I. (2006). Alfonso L. Herrera: controversia y debates durante el inicio de la biología en México. Rev. Historia Mexicana, 3. 973-1013. Recuperado de: https://www.redalyc.org/articulo.oa?id=600/60055306

De la Paz, L. J. M. (2010). De la historia natural a la profesionalización de la biología en México (1900-1940). (Tesis de maestría) Instituto Politécnico Nacional - Centro de Investigaciones Económicas, Administrativas y Sociales, México. 81-82

De la Paz, L. J. M. (2012) ¿Cómo surgió la Biología como profesión? Avances en Historia y Estudios Sociales sobre la Ciencia y la Tecnología. Trabajos en la SMHCT. Primera edición. Sociedad Mexicana de Historia de la Ciencia y de la Tecnología A.C. 224238

Domínguez-Martínez, R. (1999). La creación de la Facultad de Ciencias. Rev. Ciencias, Universidad Nacional Autónoma de México. 53. 4-13.

Ducoing, W. P. y Landesmam, S. M. (1996) Sujetos de la educación y formación docente. La investigación educativa en los ochenta, perspectivas para los noventa. Consejo Mexicano de Investigación Educativa, México. 361 pp.

Eisenberg, W. R., Olivares, S. E., Duarte, T. V., y González, D. M. (2004). El manejo del conflicto en la formación valoral ambiental del biólogo desde la investigación-acción participativa (IAP). Revista Latinoamericana de Estudios Educativos, 34(1), 85-112.

Fernández, C. A. y Lara, G. J. D. (2015). Representación social del "Modelo de desarrollo" entre biólogos y carreras afines al medio ambiente. En M. I. Ledezma y A. M. Contreras (Comps.), La Biología y los biólogos en México. Historia y representaciones sociales ( $1^{\mathrm{a}} \mathrm{ed}$.) (pp. 107-145). D.F., México: Universidad Nacional Autónoma de México.

Foliari, R., y Soms, E. (1981). Crítica al modelo teórico de la departamentalización. Revista de la Educación Superior. México. 37, 1-11.

Gaxiola, C. M. G. (1986). Historia de la biología en México en el siglo XX: La obra de Enrique Beltrán. (Tesis de licenciatura), Facultad de Ciencias. Universidad Nacional Autónoma de México. 
Gleason, R. G., López de la Rosa, L. M., y Mendoza, R. M. (1983). Diagnóstico preliminar sobre las actividades profesionales que desarrolla el biólogo en la Ciudad Universitaria. Revista de la Educación Superior, 47, 1-11.

Gómez-Luna, E., Fernando, N. D., Aponte, M. G. y Betancourt, B. L. E. (2014). Metodología para la revisión bibliográfica y la gestión de información de temas científicos, a través de su estructuración y sistematización. Dyna, 81(184),158-163. Recuperado de: https://www.redalyc.org/articulo.oa?id=496/49630405022

Granillo, V. Ma. del P., Medina, S. J., García, S. N. G., y Chino, S. S. Revisión preliminar del proceso de titulación de los egresados de la carrera de biología de la ENEP-I, UNAM. Acerca de los Temas y Áreas de los Trabajos de Tesis y de las Instituciones y Asesores Participantes. Revista de la Educación Superior. ANUIES. México. 82, 30 .

Herrera, A. L. 1939. La primitiva Sociedad Mexicana de Historia Natural. Revista de la Sociedad Mexicana de Historia Natural. 1(1): 7-14.

Hernández-Rodríguez, M., y Ruiz, G. R. (2000). Kuhn y el aprendizaje del evolucionismo biológico. Perfiles Educativos, 22(90), 92-114.

Hoffmann, A., Cifuentes, L. J. L., y Llorente-Bousquets, J. (1993). Historia del Departamento de Biología de la Facultad de Ciencias, UNAM. D.F., México: UNAM.

Islas, G. S. (1993). Pasado y presente en la formación profesional del biólogo. Perfiles Educativos. UNAM. 59, 45-54.

Jiménez, B. A. (2006). El estado del arte en la investigación en ciencias sociales. Bogotá. Universidad Pedagógica Nacional. Colombia. 27-42. Recuperado de http://biblioteca.clacso.edu.ar/Colombia/dcs-upn/20121130050742/estado.pdf

Jiménez, V. M. S. (2009). Trayectorias laborales y movilidad de los biólogos agropecuarios de la Universidad Autónoma de Tlaxcala. Perfiles educativos. UNAM. 31(126), 5679.

Jiménez, V. M. S. y Orozco, B. M. (2011) Inserción y desempeño profesional de biólogos agropecuarios de la Universidad Autónoma de Tlaxcala. Un estudio desde los empleadores y los egresados En H. Casanova Cardiel (Presidente) XI Congreso Nacional de Investigación Educativa, Nuevo León, México.

Leal, L. L. (1985). Propuesta metodológica para la evaluación del plan de estudios de la carrera de biólogo en la Facultad de Ciencias Biológicas de la Universidad Autónoma de Nuevo León, a partir de un enfoque macroeducacional. (Tesis de Maestría). Universidad Autónoma de Nuevo León.

Revista RedCA octubre 2020 - enero 2021 ISSN: 2594-2824 Vol. 3 Núm. 8 
Ledesma, M. I. (1990). Esbozo histórico de la Biología en Puebla. Quipu, Revista Latinoamericana de Historia de la Ciencia y de la Tecnología. México. 7, (1), 93125

Ledesma, M. I. (1993). Biología: ¿ciencia o naturalismo? Revista Ciencia y Desarrollo, México, D.F. XIX (110),70-77.

Ledesma, M. I. (1999). El conflicto entre Alfonso L. Herrera e Isaac Ochoterena y la Institucionalización de la Biología en México, (Tesis doctoral). Facultad de Ciencias, Universidad Nacional Autónoma de México.

Ledesma, M. I. (2006). Las tesis de biólogo en la UNAM y en la ENCB del IPN (1947-1979): un estudio comparativo con el sistema Réseau-lu”. En: Saldaña González, Juan José y Guadalupe Urbán Martínez (Eds.) Memorias del X Congreso Mexicano de Historia de la Ciencia y la Tecnología. México.

Ledesma, M. I. (2007). De Balderas a la Casa del Lago. La institucionalización de la Biología en México. Universidad Autónoma de la Ciudad de México.

Ledesma, M. I. (2009). Biología, institución y profesión: centros y periferias. México, D.F, México. Educación y Cultura.

Ledesma, M. I. (2013). La Biología y los biólogos en México: ciencia, disciplina y profesión. En M. Kleiche-Dray, J. Zubieta y M. L. Rodríguez (Coords.) La institucionalización de las disciplinas científicas en México. Siglos XVIII, XIX y XX: Estudios de caso y metodología. I.I.S. UNAM. México. 97-126.

Ledesma, M. I. (2015). La Biología y los biólogos: un esbozo histórico que se orienta a lo social. En I. Ledezma y M. Contreras Alvarado (Comps.) (2015). La Biología y los biólogos en México. Historia y representaciones sociales ( $1^{\mathrm{a}}$ ed.). D.F., México: UNAM. 9-20.

Ledesma, M. I. y Barahona, E. A. (1999). Alfonso Luis Herrera e Isaac Ochoterena: La institucionalización de la Biología en México. Rev. Historia Mexicana. El Colegio de México. 47, 635-674

Ledesma, M. I. y Barahona, E. A. (2003). The Institutionalization of Biology in Mexico in the early 20 th Century. The Conflict Between Alfonso Luis Herrera (1868-1942) and Isaac Ochoterena (1885-1950). Journal of the History of Biology. Switzerland 36 (2), 285-307.

Ledesma, M. I., y Contreras Alvarado, M. (Comps.) (2015). La Biología y los biólogos en México. Historia y representaciones sociales. D.F., México. UNAM.

Revista RedCA octubre 2020 - enero 2021 ISSN: 2594-2824 Vol. 3 Núm. 8 
López De la Rosa, L. M. (1984). Las actividades profesionales del biólogo en La Paz, Baja California Sur. Revista de la Educación Superior ANUIES, 51. Recuperado de http://publicaciones.anuies.mx/pdfs/revista/Revista51_S1A2ES.pdf

López De la Rosa, L. M. (1985). Actividades profesionales del biólogo en el Instituto Nacional de Investigaciones sobre Recursos Bióticos, en sus sedes de Jalapa y el Distrito Federal. Revista de la Educación Superior ANUIES, 54. Recuperado de http://publicaciones.anuies.mx/pdfs/revista/Revista54_S2A1ES.pdf

López De la Rosa, L. M. (1987). Actividad profesional del Biólogo en el Instituto Tecnológico y de Estudios Superiores de Monterrey y en la Facultad de Ciencias Biológicas de la Universidad Autónoma de México. Revista de la Educación Superior ANUIES, 63, Recuperado de http://publicaciones.anuies.mx/pdfs/revista/Revista63_S1A4ES.pdf

López-Trujillo (2009). La Licenciatura de Biología en México. (Tesis de doctoral). Universidad nacional Autónoma de México. 277 pp.

López-Trujillo, A., A., Nava, M. E. y Moreno, R. (2013). Exploración de los estilos de aprendizaje en los estudiantes de la carrera de biología. Revista Estilos de Aprendizaje, Universidad Complutense de Madrid, España. 6 (11), 118-138.

López-Trujillo, A., Ledesma, M.I., Cifuentes, L. J. L., Vargas, M. R., Salcedo, A. M. y Roberto Moreno, C. R. La Carrera de Biología en México. Conjunción de perspectivas. Asociación Mexicana de Facultades y Escuelas de Biología A.C., México.

López-Trujillo, A., y Saucedo, M. (1999). La licenciatura en Biología en la ENEP - Iztacala de la. UNAM. Revista de la Educación Superior, ANUIES. 109, 1-22. Recuperado de http://publicaciones.anuies.mx/pdfs/revista/Revista109_S2A3ES.pdf

Lugo, V. E. (2003a). El currículum flexible: la opinión de los estudiantes de cuatro universidades públicas. En Zorrilla, F. M. (Presidenta) Memorias del VII Congreso Nacional de Investigación Educativa, COMIE, Guadalajara, Jalisco. México

Lugo, V. E. (2003b). Estudio Comparativo de Modelos Curriculares Flexibles. (Tesis doctoral). Universidad Autónoma del Estado de Morelos, Morelos, México.

Matus-Parada, J. (2011). Problemas y alternativas de aprendizaje de una actividad compleja en la formación del biólogo. Revista Mexicana de Psicología Educativa (RMPE). México. 2 (1), 39-50.

Medina, R.J. S. y Ledesma, M. I. (2015). Entre lo sociológico y lo histórico: elementos de la representación social de la evolución entre estudiantes de Biología, Medicina y Psicología. En M. I. Ledezma y A. M. Contreras (Comps.), La Biología y los biólogos

Revista RedCA octubre 2020 - enero 2021 ISSN: 2594-2824 Vol. 3 Núm. 8 
en México. Historia y representaciones sociales ( $1^{\mathrm{a}}$ ed.) (pp. 91-105). D.F., México: Universidad Nacional Autónoma de México.

Méndez, R. L. M. (1985). Las actividades profesionales del biólogo en diversas dependencias de la secretaria de agricultura y recursos hidráulicos. Revista de la Educación Superior, ANUIES. 55, $17 . \quad$ Recuperado de http://publicaciones.anuies.mx/revista/55/1/2/es/las-actividades-profesionales-delbiologo-en-diversas-dependencias-de

Millán, P., Carmona, P. R., y Zárate, B. (1997). Comprensión del concepto de selección natural en estudiantes universitarios. Revista Mexicana de Investigación Educativa, ANUIES. II (3), 1-19. Recuperado de http://www.redalyc.org/pdf/140/14000304.pdf

Monterrosas, B. M. (2010). La tutoría como un dispositivo que integre perspectivas de estudiantes, docentes, directivos, currícula y gestión educativa (Tesis de maestría). Universidad Pedagógica Nacional Morelos, Cuernavaca, Morelos, México.

Monterrosas, B. M. (2018). Establecimiento del vínculo tutor-tutorado y su repercusión en el proceso de formación profesional de los estudiantes (Tesis doctoral). Universidad Pedagógica Nacional Morelos, Cuernavaca, Morelos. México.

Noriega, L. M. D., Lagunas, H. C. A. y Recio, U. C. A. (2009) Políticas públicas y educación superior en México. X Congreso Nacional de Investigación. En R. López Zárate (Presidente) X Congreso Nacional de Investigación Educativa. Veracruz, México. Recuperado de http://xplora.ajusco.upn.mx:8080/xplora-pdf/0508-F.pdf

Ordoñez, F. (2009). El currículo Flexible en la Universidad Juárez Autónoma de Tabasco: los primeros momentos. El caso de la División Académica de Ciencias Biológicas. En R. López Zárate (Presidente), X Congreso Nacional de Investigación Educativa, COMIE, Veracruz, México.

Ortega, M. M., Godínez, J.L. y Vilaclara, G. (1996). Relación histórica de los antecedentes y origen del Instituto de Biología de la UNAM. Universidad Nacional Autónoma de México. 98 p.

Páez, B. (1970). Estudio del Mercado de Trabajo del Biólogo. Informe Final. Instituto de Investigaciones Industriales. Monterrey, N. L., México.

Peña, V. T. y Pirela, M. J. (2007). La complejidad del análisis documental. Información, Cultura y Sociedad. Buenos Aires. (16), 55-81.

Pino, F. A. (2008). La relación entre docencia e investigación. El caso del departamento de biología de la Facultad de Ciencias de la UNAM. Rev. Andamios. Universidad Autónoma de la Ciudad de México. 5 (9), 205-239 Recuperado de: https://www.redalyc.org/articulo.oa? $\mathrm{id}=62811466010$

Revista RedCA octubre 2020-enero 2021 ISSN: 2594-2824 Vol. 3 Núm. 8 
Rebolledo, L. M.A. y Ayala, R. M. (2006) Evaluación del desempeño docente del Departamento de Biología Marina de la Universidad Autónoma de Baja california Sur. México Revista Latinoamericana de Estudios Educativos. México. XXXVI (1-2), 95-133. Recuperado de: https://www.redalyc.org/articulo.oa?id=270/27036207

Rebolledo, L. Ma. A., Gómez del Prado, M.C. R., y Romero V.E. (2005). Caracterización de los estudiantes de la carrera de Biólogo Marino de la UABCS. En Zorrilla F. M. (Presidenta) II Congreso Nacional de Investigación Educativa COMIE, Hermosillo, Sonora, México.

Rebolledo, L. A., y Romero V. E. (2011). El estudiante de biología marina de la UABCS. Relación entre sus características al ingreso y su trayectoria escolar. En H. Casanova Cardiel (Presidente) XI Congreso Nacional de Investigación Educativa, Nuevo León, México.

Reyes, L.A., Reyes, M. M. y Pérez-Bonilla, M. E. (2016). Experimentación virtual con el simulador dosis-respuesta como herramienta docente en biología. Revista Apertura. Guadalajara, Jal, México. 8 (2), 22-37. Recuperado de: http://www.scielo.org.mx/scielo.php?script=sci_arttext\&pid=S1665$61802016000300022 \& \operatorname{lng}=$ es\&tlng=es.

Ríos Celis, I., López de La Rosa, L. M. y Mendoza, R. M. (1986). Elementos para el seguimiento académico en la carrera de biología de la Facultad de Ciencias de la UNAM. Revista de la Educación Superior, 15(57). Recuperado de http://publicaciones.anuies.mx/pdfs/revista/Revista57_S2A4ES.pdf

Rodríguez, J. M. (1987). La educación superior de la biología en México. Facultad de Ciencias-UNAM. México.

Romero, V. E., Rebolledo, L. A., y Gómez del Prado, R. M. del C. (2007). Trayectoria escolar de los estudiantes de la carrera de biólogo marino de la UABCS y su vinculación con el nivel medio superior del estado de BCS. En R. López Zárate (Presidente) $X$ Congreso Nacional de Investigación Educativa. COMIE, México.

Sigala, R. J. (2014). María de los Dolores Barba Ávila. Ernestina Meza Rangel. Guadalupe Castorena Esparza. Gabriel González Adame (Coordinadores). La biología en la UAA: 40 años de desarrollo. Universidad Autónoma de Aguascalientes. México. 229.

Tirado, S. F. y López-Trujillo, A. (1994). Problemas de la enseñanza de la biología en México. Perfiles Educativos, UNAM. 66, 51-57.

Toledo, V. M., Las 4 Biologías de una universidad subdesarrollada: La UNAM. Revista Biología, 1-4 (5): 1975. 
Torres-Ochoa, S. R. (2002). La investigación Científica en la biología y su impacto en la docencia universitaria. El caso de la universidad Michoacana. (Tesis doctoral). Escuela Nacional de Estudios Profesionales, Acatlán, UNAM. México.

Torres-Ochoa, S. R. (2006). Identidad formativa en la educación superior. El caso de biología. Revista de la educación superior. México. 35(139), 49-65. Recuperado de: http://www.scielo.org.mx/scielo.php?script=sci_arttext\&pid=S018527602006000300049\&lng=es\&tlng=. Pdf

Torres Ochoa, S. R. (2011). Cognición en conceptos básicos en microbiología para estudiantes de Biología. En M. Rosa Buxarrais (Presidenta) XII Congreso Internacional de Teoría de la Educación. Barcelona, España.

Urquidi, P. (2011). El sentido de los estudios y las prácticas de los estudiantes universitarios (Tesis de maestría). Instituto de Investigaciones en Educación, Universidad Veracruzana, México.

Vickery, B. (1970). Techniques of information retrieval. Londres: Butterworths.

Weiss, E. (2003). Usos de la investigación educativa. Revista Mexicana de Investigación Educativa. México. 8 (19), 597-598. 